\title{
Effect of 5-alpha Reductase Inhibitor on Storage Symptoms in Patients with Benign Prostatic Hyperplasia
}

\author{
Kang Jun Cho, Se Hee Kang, Hyo Sin Kim, Jun Sung Koh, Joon Chul Kim \\ Department of Urology, The Catholic University of Korea School of Medicine, Seoul, Korea
}

Purpose: Many patients with benign prostatic hyperplasia (BPH) have storage symptoms. The aim of this study was to evaluate the effects of treatment with a 5 -alpha reductase inhibitor (5ARI) on storage symptoms in patients with BPH.

Methods: This study was conducted in 738 patients with lower urinary tract symptoms secondary to BPH. Patients with a prostate volume of higher than $30 \mathrm{~mL}$ on the transrectal ultrasound were classified into two groups: group A, in which an alpha blocker was solely administered for at least 12 months, and group B, in which a combination treatment regimen of an alpha blocker plus 5ARI was used. This was followed by an analysis of the changes in parameters such as the total International Prostate Symptom Score (IPSS), voiding symptom subscore, and storage symptom subscore between the two groups. In addition, we examined whether there was a significant difference between the two groups in the degree of change in storage symptoms between before and after the pharmacological treatment.

Results: Of the $738 \mathrm{men}, 331$ had a prostate volume $\geq 30 \mathrm{~mL}$, including 150 patients in group A and 181 patients in group B. Total IPSS, the voiding symptom subscore, and the storage symptom subscore were significantly lower after treatment than before treatment in both groups $(\mathrm{P}<0.05)$. A comparison of the degree of change between before and after treatment, however, showed no significant differences in the storage symptom subscore between the two groups $(\mathrm{P}>0.05)$.

Conclusions: Alpha blocker and 5ARI combination treatment is effective for patients with BPH including storage symptoms. However, 5ARI does not exert a significant effect on storage symptoms in BPH patients.

Keywords: Prostatic hyperplasia; 5-alpha reductase inhibitors; Overactive urinary bladder

\section{INTRODUCTION}

Benign prostatic hyperplasia $(\mathrm{BPH})$ is a disease that causes bladder outlet obstruction (BOO) resulting from functional obstruction due to the adjustment of the nerves innervating the smooth muscle layer of the prostate gland and anatomical obstruction due to the increased size of the prostate gland. $\mathrm{BPH}$ is one of the main etiologic factors responsible for the occurrence of lower urinary tract symptoms (LUTS) in post-middle aged men [1]. The pharmacological treatments for BPH mainly include alpha blockers and 5-alpha reductase inhibitors (5ARIs). 5ARI blocks the pathway through which testosterone is transformed into dehydrotestosterone [2] and thereby suppresses the proliferation of interstitial cells and epithelial cells in the prostate gland. It is also known to reduce the size of the prostate gland by apoptosis in the prostate gland tissue [3]. The LUTS of $\mathrm{BPH}$, the severity of which is improved by these pharmacological treatments, can be divided into voiding symptoms and storage symptoms. The alpha blockers and 5ARIs that are currently available have focused on the alleviation of voiding symptoms. Of the LUTS, however, storage symptoms are more common than voiding symptoms [4] and are known to have a greater effect on quality of life (QoL) [5,6]. In the treatment of $\mathrm{BPH}$, therefore, the effect on storage symptoms should be seriously considered. The storage symptoms that patients with BPH complain of are closely associated with the detrusor muscle as a sec-
Corresponding author: Joon Chul Kim

Department of Urology, Bucheon St. Mary's Hospital, The Catholic University of Korea School of Medicine, 2 Sosa-dong, Wonmi-gu, Bucheon 420-717, Korea Tel: +82-32-340-7071 / Fax: +82-32-340-2124 / E-mail: kjc@catholic.ac.kr Submitted: August 7, 2011 / Accepted after revision: September 6, 2011
This is an Open Access article distributed under the terms of the Creative Commons Attribution Non-Commercial License (http://creativecommons.org/licenses/by-nc/3.0/) which permits unrestricted non-commercial use, distribution, and reproduction in any medium, provided the original work is properly cited. 
ondary change due to the $\mathrm{BOO}$ [7]. Treatment agents for $\mathrm{BPH}$ alleviate this $\mathrm{BOO}$ and thereby improve the voiding symptoms. There is also a possibility, therefore, that they might have an effect on the storage symptoms. As a matter of fact, little is known about the exact pharmacological mechanisms. On the basis of various hypotheses, the effects of alpha blockers on storage symptoms have been reported, but to the effects of 5ARI have not yet been investigated.

The International Prostate Symptom Score (IPSS) is commonly used to assess the initial symptoms of patients with $\mathrm{BPH}$, and changes in this score have been reported to be useful in assessing the treatment response or the disease progression. We speculated that the IPSS would be a useful indicator for the current study because it is divided into a voiding symptom subscore and a storage symptom subscore.

Given the above background, we attempted to examine the effect of 5ARI on storage symptoms in BPH patients. We compared the difference in changes in storage symptoms between an alpha blocker monotherapy group and an alpha blocker+ 5ARI combination therapy group with the use of the IPSS.

\section{MATERIALS AND METHODS}

The current study was a single-center, retrospective analysis and it was approved by the institutional review board of our medical institution. The current study was conducted in 738 patients who visited the outpatient clinic with a chief complaint of LUTS during a period ranging from January 2008 to March 2009. All patients underwent tests such as urinalysis, prostate-specific antigen (PSA) measurement, maximal uroflow rate (Qmax), postvoid residual volume (PVR), transrectal ultrasonography (TRUS), and completion of the IPSS questionnaire.

Patients with LUTS (IPSS $\geq 8$ points) and treated with an alpha blocker (e.g., doxazosin, tamsulosin, terazosin, or alfuzo$\sin$ ) and/or 5ARI (e.g., finasteride or dutasteride) for at least 12 months and with a prostate volume of higher than $30 \mathrm{~mL}$ on the TRUS were enrolled. Exclusion criteria were patients who were diagnosed with prostate cancer following the test, who underwent transurethral resection of the prostate or other surgical intervention related to $\mathrm{BPH}$, who had neurogenic bladder, or who had a history of recurrent or complicated urinary tract infection or bladder stones. Enrolled patients were classified into two groups: group A, in which the alpha blocker was solely administered for at least 12 months, and group B, in which a combination treatment regimen of alpha blocker with 5ARI was used for at least 12 months. The patients took alpha blocker and 5ARI from the beginning of treatment; the 5ARI was not added after alpha blocker monotherapy. This was followed by an analysis of the changes in parameters such as the total IPSS, voiding symptom subscore, storage symptom subscore, and QoL score between the two groups. In addition, we examined whether there was a significant difference between the two groups in the degree of change in storage symptoms between before and after the pharmacological treatment. Statistical analysis was performed by using paired Student's t-tests. All data were analyzed by using SPSS ver. 17.0 (SPSS Inc., Chicago, IL, USA). A value of $\mathrm{P}<0.05$ was considered statistically significant.

\section{RESULTS}

Of the $738 \mathrm{men}, 331$ (44.8\%) had a prostate volume of $\geq 30 \mathrm{~mL}$ and had been treated with medication for at least 12 months. A total of 150 patients had been treated with an alpha blocker only (group A) and 181 patients had received combined medication with an alpha blocker and 5ARI (group B). The mean age of the patients was $62.4 \pm 0.82$ years in group $A$ and $67.08 \pm 0.60$ years in group $\mathrm{B}$. The prostate volume was $41.5 \pm 1.73 \mathrm{~mL}$ on average in group $\mathrm{A}$ and $48.1 \pm 1.38 \mathrm{~mL}$ on average in group B. Before treatment, there were no significant differences in Qmax, PSA, or PVR between the two groups $(\mathrm{P}>0.05)$.

Before treatment, the IPSS and voiding symptom subscore were $14.65 \pm 0.67$ points and $8.42 \pm 0.46$ points in group A, respectively, and $16.46 \pm 0.67$ points and $9.61 \pm 0.44$ points in group $B$, respectively. This difference was not statistically significant $(\mathrm{P}>0.05)$ (Table 1). After treatment, the IPSS and voiding symptom subscore were $12.16 \pm 0.68$ points and $6.82 \pm 0.44$ points in group A (Fig. 1), respectively, and $13.77 \pm 0.69$ points and $7.93 \pm 0.41$ points in group B (Fig. 2), respectively. These results indicated that the IPSS and voiding symptom subscore were significantly lower after treatment than before treatment $(\mathrm{P}<0.05)$. In addition, before treatment, the storage symptom subscore was $6.23 \pm 0.31$ points in group $A$ and $6.84 \pm 0.31$ points in group $\mathrm{B}$. This difference was not statistically significant $(\mathrm{P}>$ $0.05)$. After treatment, it was found to be $5.34 \pm 0.29$ points and $5.83 \pm 0.31$ points in groups $A$ and $B$, respectively. These results indicate that the storage symptom subscore was significantly lower after treatment than before in both groups $(\mathrm{P}<0.05)$.

Before treatment, the QoL score was $3.47 \pm 0.10$ points in group A and $3.71 \pm 0.09$ points in group B. This difference was not statistically significant $(P>0.05)$. After treatment, the QoL 
Table 1. Comparison of the baseline characteristics of the patients

\begin{tabular}{lccc}
\hline Variables & Group A & Group B & P-value \\
\hline No. of patients & 150 & 181 & \\
Age $(\mathrm{yr})$ & $62.40 \pm 0.82$ & $67.08 \pm 0.60$ & 0.001 \\
Prostate volume $(\mathrm{g})$ & $41.50 \pm 1.73$ & $48.10 \pm 1.38$ & 0.007 \\
Qmax $(\mathrm{mL} / \mathrm{sec})$ & $12.73 \pm 0.65$ & $11.95 \pm 0.40$ & 0.549 \\
PSA $(\mathrm{ng} / \mathrm{mL})$ & $3.33 \pm 0.44$ & $4.21 \pm 0.50$ & 0.210 \\
PVR $(\mathrm{mL})$ & $59.0 \pm 9.71$ & $86.43 \pm 7.40$ & 0.17 \\
IPSS & & & \\
Total & $14.65 \pm 0.67$ & $16.46 \pm 0.67$ & 0.061 \\
Storage subscore & $6.23 \pm 0.31$ & $6.84 \pm 0.31$ & 0.172 \\
Voiding subscore & $8.42 \pm 0.46$ & $9.61 \pm 0.44$ & 0.066 \\
QoL subscore & $3.47 \pm 0.106$ & $3.71 \pm 0.094$ & 0.099 \\
\hline
\end{tabular}

Values are presented as mean \pm SEM.

Qmax, maximum urinary flow rate; PSA, prostate specific antigen; PVR, post voiding residual volume; IPSS, International Prostate Symptom Score ; QoL, quality of life.

Group A: alpha blocker group. Group B: alpha blocker + 5ARI group.

score was $2.90 \pm 0.11$ points and $3.09 \pm 0.10$ points in groups $A$ and $\mathrm{B}$, respectively. These results indicate that the QoL score was significantly lower after treatment than before in both groups $(\mathrm{P}<0.05)$.

The comparison of the degree of change between before and after treatment, however, showed that there were no significant differences in the storage symptom subscore between the two groups $(\mathrm{P}>0.05)$ (Fig. 3).

\section{DISCUSSION}

In the current study, the analysis of the baseline characteristics of the patients showed that mean age and mean prostate volume were relatively higher in group B than in group A. According to several BPH guidelines, combination therapy with an alpha blocker and 5ARI is more effective in patients with symptoms related to a large prostate volume; therefore, concomitant use of these treatment regimens has been recommended $[8,9]$. According to one study that examined the degree of use of combination therapy in an actual clinical setting, the use was proportional to the size of prostate gland [10]. Concomitant use of the two treatment regimens is prescribed by physicians with a complete understanding of the above rationale. It can therefore be inferred that the size of the prostate gland was slightly greater in the combination therapy group than in the monotherapy group.

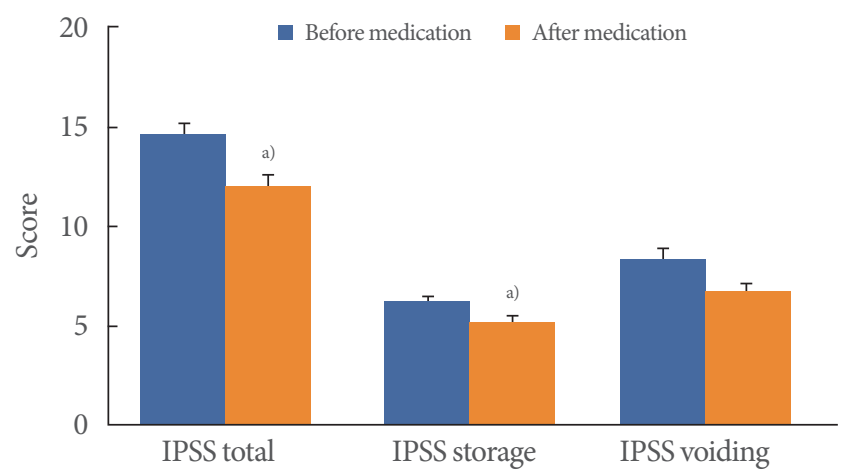

Fig. 1. Comparison of the total International Prostate Symptom Score (IPSS) and the storage and voiding subscores between before and after medication (month 12) in group A (alpha blocker group). ${ }^{\text {a) }} \mathrm{P}<0.05$ compared with before medication.

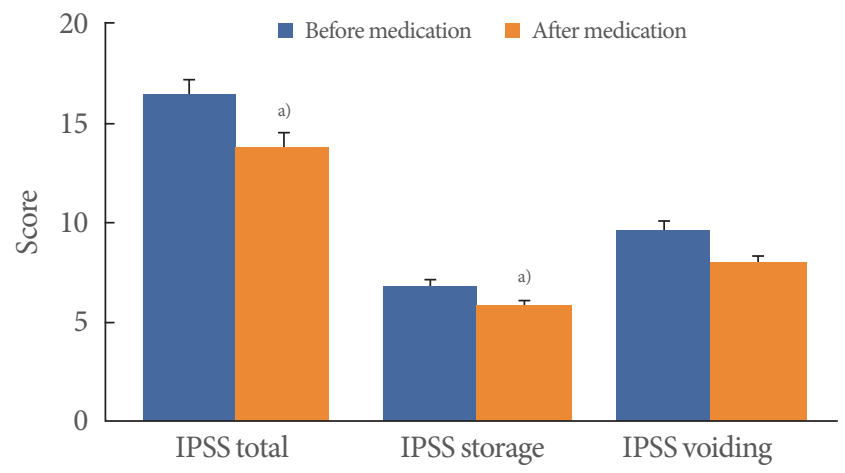

Fig. 2. Comparison of the total International Prostate Symptom Score (IPSS) and the storage and voiding subscores between before and after medication (month 12) in group B (alpha blocker +5 -alpha reductase inhibitor group). ${ }^{\text {a) }} \mathrm{P}<0.05$ compared with before medication.

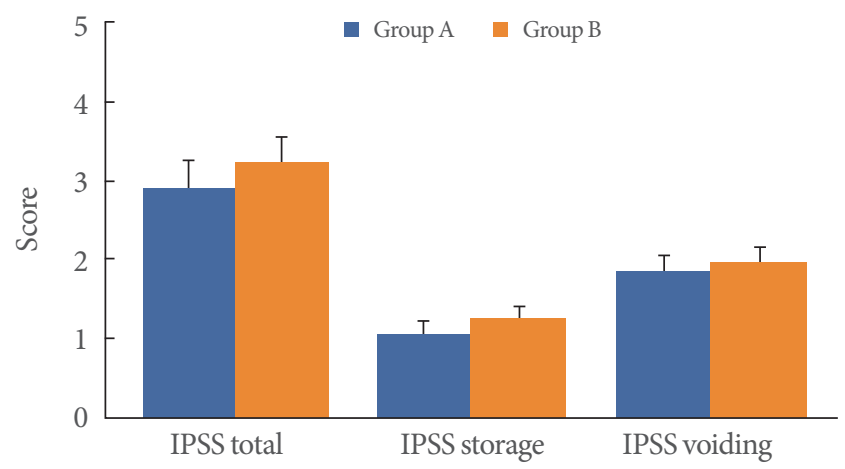

Fig. 3. The mean change from baseline to 12 months in the total International Prostate Symptom Score (IPSS) and the voiding and storage subscores in group A (alpha blocker group) and group B (alpha blocker + 5-alpha reductase inhibitor group). 
It is well established that the size of the prostate gland increases with age. This leads to the speculation that the mean age was higher in group B, in which the size of the prostate gland was relatively greater than in group $\mathrm{A}$. It is also probable that the difference in these baseline characteristics of the patients might have affected the treatment outcomes. However, according to the measures indicative of the symptoms, such as the IPSS, Qmax, and PVR, there were no significant differences between the two groups. This implies that age and prostate volume were not significant pretreatment factors that affected the treatment outcomes.

The IPSS, which was used as an indicator of the treatment outcomes in the current study, has already been reported in other studies to have no relationship with prostate volume [11]. It is therefore presumed that the difference in the prostate volume might not have been a significant factor determining treatment outcomes. In the current study, the total IPSS, voiding symptom subscore, and storage symptom subscore were significantly decreased after the drug treatments in both groups. Several studies have reported the effects of alpha blocker on storage symptoms. A long time ago, Lepor et al. [12] reported that the total IPSS, voiding symptom subscore, and storage symptom subscore were significantly decreased after the use of terazosin. Recent studies have examined naftopidil, an alpha 1A/D adrenoceptor blocker, and showed that the voiding symptom subscore and storage symptom subscore were significantly decreased after monotherapy with naftopidil. In addition, after the monotherapy with naftopidil, the symptoms of nocturia were also improved in patients with BPH who concurrently had nocturnal polyuria [13]. Furthermore, according to a study using silodosin, an alpha $1 \mathrm{~A}$ adrenoceptor selective antagonist, the total IPSS and storage and voiding symptom subscores were significantly decreased, the maximum cystometric capacity was significantly increased, and the detrusor overactivity was lost and then improved. These findings were also demonstrated in the urodynamic study [14].

Little is known about the exact mechanisms by which alpha blockers affect storage symptoms. Nevertheless, the following hypotheses exist: an alpha $1 \mathrm{D}$ receptor is involved in the regulation of the detrusor overactivity, because it is mainly distributed in the bladder detrusor muscle, and the micturition reflex is suppressed owing to the blockage of alpha 1D receptor, which is distributed in the spinal cord nerve system. Moreover, animal experiments have demonstrated that an alpha $1 \mathrm{~A}$ receptor is involved in the c-fiber afferent activity. This hypothesis led to the reports showing that alpha blockers improve the storage symptom in patients with $\mathrm{BPH}[15,16]$. Another hypothesis is that the bladder outlet resistance is decreased if the size of the enlarged prostate, which mechanically obstructs the bladder outlet, is decreased in patients with BPH, thus leading to an improvement in storage symptoms. On the basis of this concept, Becher et al. [17] reported that storage symptoms were improved with the use of dutasteride, a type of $5 \mathrm{ARI}$, alone, and that in the dutasteride and tamsulosin combination treatment group, as compared with the monotherapy group, the storage and voiding subscores were significantly decreased. In addition, the voiding subscore was further decreased.

In another study, an analysis was performed mainly to evaluate the effects on storage symptoms of a concomitant treatment regimen of finasteride with alpha blocker. That study showed effects of a markedly higher degree in improving the symptoms in patients with severe storage symptoms [18]. We investigated the effect in $\mathrm{BPH}$ patients of medical treatment of more than 12 months. Reduction of prostate volume by $5 \mathrm{ARI}$ was noted from 3 months markedly and was sustained for 12 months, but the degree of reduction after 1 year was not significant [19]. In the current study, as shown in other studies, a comparison was made between the combination therapy group and the monotherapy group. Through a comparative analysis of whether there was a significant difference in the degree of improvement in symptoms between the two groups from the perspective of storage symptoms, the effect of 5ARI on storage symptoms was confirmed. In the current study, there were also effects in improving the storage symptoms in the combination therapy group. In the comparison of the degree of the improvement in the storage symptoms between the monotherapy group and the combination therapy group, however, no significant differences were found. These results lead us to speculate that 5ARI has an equivalent profile or a lower degree of effect on the storage symptoms as compared with alpha blocker. This might be because although 5ARI improves the BOO in a manner similar to that of alpha blocker, the mechanism may be one of the major mechanisms by which alpha blocker improves storage symptoms, such as the inhibition of the micturition reflex in the bladder or spinal cord. This suggests that although 5ARI is clinically useful from the perspective of voiding symptoms because it improves the incidence of acute urinary retention, BPH-related surgery, and the peak urinary flow rate, it might not be of great help in improving storage symptoms. It can therefore be inferred that the additional use of $5 \mathrm{ARI}$ might not be of further help in patients who 
complain of a persistent presence of storage symptoms and have a past history of taking an alpha blocker. Thus, additional methods by which the storage symptoms can be actively treated should be considered.

Although patients were not additionally given anticholinergics in the current study, several studies have reported that a combination treatment regimen of alpha blocker with anticholinergics has a sufficient degree of effect on storage symptoms and produces no side effects in patients with BPH [20,21]. It can therefore be concluded that concomitant treatment with other drugs such as anticholinergics should be considered. Additionally, the IPSS used in the current study has been demonstrated to be effective in evaluating the treatment response. To clarify this more objectively, however, objective test regimens such as an urodynamic study should be performed in patients who complain of storage symptoms and in whom 5ARI was solely administered.

Another limitation of our study is that we did not analyze the difference by severity of storage symptoms. In the CombAT study [22], the mean IPSS storage subscores at baseline were 7.3 and 7.2 in the tamsulosin groups, and storage symptoms improved significantly in the patients who received combination therapy compared with monotherapy. In our study, the mean IPSS storage subscores at baseline were 6.84 in the combination therapy group and 6.23 in the alpha blocker therapy group. Thus, the mean IPSS storage subscores at baseline were less in our study than in the CombAT study. A difference in treatment results by severity of storage symptoms might have existed, and we should consider this aspect in the future.

\section{CONFLICT OF INTEREST}

No potential conflicts of interest relevant to this article are reported.

\section{REFERENCES}

1. Chapple CR. Pharmacological therapy of benign prostatic hyperplasia/lower urinary tract symptoms: an overview for the practising clinician. BJU Int 2004;94:738-44.

2. McConnell JD. The pathophysiology of benign prostatic hyperplasia. J Androl 1991;12:356-63.

3. Glassman DT, Chon JK, Borkowski A, Jacobs SC, Kyprianou N. Combined effect of terazosin and finasteride on apoptosis, cell proliferation, and transforming growth factor-beta expression in be- nign prostatic hyperplasia. Prostate 2001;46:45-51.

4. Irwin DE, Milsom I, Hunskaar S, Reilly K, Kopp Z, Herschorn S, et al. Population-based survey of urinary incontinence, overactive bladder, and other lower urinary tract symptoms in five countries: results of the EPIC study. Eur Urol 2006;50:1306-14.

5. Djavan B. Lower urinary tract symptoms/benign prostatic hyperplasia: fast control of the patient's quality of life. Urology 2003;62(3 Suppl 1):6-14.

6. Roehrborn CG. Current medical therapies for men with lower urinary tract symptoms and benign prostatic hyperplasia: achievements and limitations. Rev Urol 2008;10:14-25.

7. Mirone V, Imbimbo C, Longo N, Fusco F. The detrusor muscle: an innocent victim of bladder outlet obstruction. Eur Urol 2007;51:5766.

8. AUA Practice Guidelines Committee. AUA guideline on management of benign prostatic hyperplasia (2003). Chapter 1: Diagnosis and treatment recommendations. J Urol 2003;170(2 Pt 1):530-47.

9. Madersbacher S, Alivizatos G, Nordling J, Sanz CR, Emberton M, de la Rosette JJ. EAU 2004 guidelines on assessment, therapy and follow-up of men with lower urinary tract symptoms suggestive of benign prostatic obstruction (BPH guidelines). Eur Urol 2004;46: 547-54.

10. Oh CY, Lee SH, Yoo SI, Chung BH. Korean urologist's view of practice patterns in diagnosis and management of benign prostatic hyperplasia: a nationwide survey. Yonsei Med J 2010;51:248-52.

11. Vesely S, Knutson T, Damber JE, Dicuio M, Dahlstrand C. Relationship between age, prostate volume, prostate-specific antigen, symptom score and uroflowmetry in men with lower urinary tract symptoms. Scand J Urol Nephrol 2003;37:322-8.

12. Lepor H, Auerbach S, Puras-Baez A, Narayan P, Soloway M, Lowe F, et al. A randomized, placebo-controlled multicenter study of the efficacy and safety of terazosin in the treatment of benign prostatic hyperplasia. J Urol 1992;148:1467-74.

13. Takahashi S, Tajima A, Matsushima H, Kawamura T, Tominaga T, Kitamura T. Clinical efficacy of an alpha1A/D-adrenoceptor blocker (naftopidil) on overactive bladder symptoms in patients with benign prostatic hyperplasia. Int J Urol 2006;13:15-20.

14. Yamanishi T, Mizuno T, Tatsumiya K, Watanabe M, Kamai T, Yoshida K. Urodynamic effects of silodosin, a new alpha 1A-adrenoceptor selective antagonist, for the treatment of benign prostatic hyperplasia. Neurourol Urodyn 2010;29:558-62.

15. Malloy BJ, Price DT, Price RR, Bienstock AM, Dole MK, Funk BL, et al. Alpha1-adrenergic receptor subtypes in human detrusor. J Urol 1998;160(3 Pt 1):937-43.

16. Yokoyama O, Ito H, Aoki Y, Oyama N, Miwa Y, Akino H. Selective 
a1A-blocker improves bladder storage function in rats via suppression of C-fiber afferent activity. World J Urol 2010;28:609-14.

17. Becher E, Roehrborn CG, Siami P, Gagnier RP, Wilson TH, Montorsi F. The effects of dutasteride, tamsulosin, and the combination on storage and voiding in men with benign prostatic hyperplasia and prostatic enlargement: 2-year results from the Combination of Avodart and Tamsulosin study. Prostate Cancer Prostatic Dis 2009; 12:369-74.

18. Lee JY, Lee SH, Kim SJ, Kim CS, Lee HM, Kim CI, et al. Change in International Prostate Symptom storage subscore after long-term medical therapy in BPH patients: finasteride and alpha-blocker combination therapy in men with moderate-to-severe LUTS/BPH in Korea. Urology 2011;77:171-6.

19. Debruyne F, Barkin J, van Erps P, Reis M, Tammela TL, Roehrborn C. Efficacy and safety of long-term treatment with the dual 5 alphareductase inhibitor dutasteride in men with symptomatic benign prostatic hyperplasia. Eur Urol 2004;46:488-94.
20. Bae JH, Kim SO, Yoo ES, Moon KH, Kyung YS, Kim HJ. Efficacy and safety of low-dose propiverine in patients with lower urinary tract symptoms/benign prostatic hyperplasia with storage symptoms: a prospective, randomized, single-blinded and multicenter clinical trial. Korean J Urol 2011;52:274-8.

21. Yang Y, Zhao XF, Li HZ, Wang W, Zhang Y, Xiao H, et al. Efficacy and safety of combined therapy with terazosin and tolteradine for patients with lower urinary tract symptoms associated with benign prostatic hyperplasia: a prospective study. Chin Med J (Engl) 2007; 120:370-4.

22. Montorsi F, Roehrborn C, Garcia-Penit J, Borre M, Roeleveld TA, Alimi JC, et al. The effects of dutasteride or tamsulosin alone and in combination on storage and voiding symptoms in men with lower urinary tract symptoms (LUTS) and benign prostatic hyperplasia (BPH): 4-year data from the Combination of Avodart and Tamsulosin (CombAT) study. BJU Int 2011;107:1426-31. 